\title{
Continuous Freezing in Three Dimensions
}

\author{
Richard P. Sear ${ }^{1}$ and Daan Frenkel ${ }^{2}$ \\ ${ }^{1}$ Department of Physics, University of Surrey, Guildford, Surrey GU2 7XH, United Kingdom \\ ${ }^{2}$ FOM Institute for Atomic and Molecular Physics, Kruislaan 407, NL-1098 SJ Amsterdam, The Netherlands
}

(Received 25 November 2002; published 15 May 2003)

\begin{abstract}
We analyze the freezing transition in a system of hard particles with a very long-ranged repulsion. The long-range repulsion makes first-order freezing transitions continuous, but leaves the initial stages of the crystallization unchanged: the crystal phase must still nucleate. The coexistence between bulk phases is replaced by microphase separation.
\end{abstract}

DOI: 10.1103/PhysRevLett.90.195701

PACS numbers: 64.70.Dv, 64.60.Qb

Hard spheres undergo a strongly first-order freezing transition once the volume fraction of the spheres exceeds 49.4\%. At that density, the hard-sphere fluid coexists with a face-centered cubic solid with volume fraction of $54.5 \%$. This transition is shifted, but not changed qualitatively by the presence of weak, attractive, interactions. The effect of weak, long-ranged attractive forces on the hard-spheres freezing transition was first discussed by Longuet-Higgins and Widom [1]. In the spirit of the van der Waals theory of the liquid-vapor transition, Longuet-Higgins and Widom used perturbation theory to compute the effect of attractive forces on the freezing transition. This approach was placed on a rigorous footing by the work of Kac et al. [2], van Kampen [3], and Lebowitz and Penrose [4]. This work showed that the form of the free energy pioneered in the 19th century by van der Waals, becomes exact for weak, long-ranged attractions, in the limit that the strength goes to zero and the range goes to infinity in such a way that the integrated strength of the potential remains finite.

In this Letter, we discuss the effect of weak, longranged repulsive forces on the freezing transition of hard spheres. At first sight, one might expect that the van der Waals approach should work just as well for weak repulsive as for weak attractive forces, but this is not the case. In fact, Lebowitz and Penrose [4] showed that there is a rather surprising asymmetry between the case of weak attractions and that of weak repulsions. In particular, they concluded that, if the same limiting procedure is applied to repulsive interactions, any preexisting phase transition will be suppressed. To quote Ref. [4]: "[such a repulsive potential] causes the distinct liquid and gas phases of a normal first-order transition to break up into droplets or froth...." Note that the discussion of Lebowitz and Penrose focuses on the liquid-vapor transition, i.e., a transition between two phases with the same symmetry.

To our knowledge, little attention has been paid to the possible implications of the Lebowitz-Penrose scenario for freezing. By applying the exact Lebowitz-Penrose perturbation theory, we find that if a repulsion with a range $\lambda$ times the hard-sphere diameter is added to the hard-sphere repulsion, the transition from fluid to crystal becomes continuous in the $\lambda \rightarrow \infty$ limit. It is continuous in the sense that although there is a discontinuity in the density derivative of the pressure, there is no plateau in the pressure itself and so no coexistence of phases with differing densities. By going beyond the theory of Lebowitz and Penrose and considering inhomogeneous systems and finite ranges $\lambda$ of the repulsion, we show that switching on a long-range repulsion breaks up coexisting fluid and crystalline phases into domains of size many times the hard-sphere diameter but much smaller than $\lambda$. Switching on the long-range repulsion has the effect of driving the solid-fluid interfacial free energy to zero. In this respect, long-range repulsions act like a surfactant. Even if the repulsion is too weak (or too short ranged) to break up coexisting phases into domains, it may significantly increase rates of nucleation, and so alter the phase-transition dynamics. However, the effect on the phase-transition dynamics is limited to not-too-large $\lambda$. In the $\lambda \rightarrow \infty$ limit nucleation is identical to that in hard spheres, resulting in the surprising situation where although the transitions are continuous, before they can occur a nucleation barrier must be surmounted.

We start by defining a suitable repulsive pair potential,

$$
u=\left\{\begin{array}{cc}
\infty, & r \leq \sigma \\
w(r), & r>\sigma,
\end{array}\right.
$$

where $w(r)$ is a Kac potential

$$
w(r)=\epsilon \lambda^{-3} \exp [-r /(\lambda \sigma)] .
$$

The choice of this form for $w(r)$ is made for the sake of convenience. For $\epsilon>0$, the long-range pair interaction is repulsive, and for $\epsilon<0$ it is attractive. The exact results of Lebowitz and Penrose apply in the $\lambda \rightarrow \infty$ limit. The contribution of $w(r)$ to the second virial coefficient is just half its integral over all space, divided by the thermal energy $k_{B} T$ [5]. We denote this contribution to the second virial coefficient by $a=4 \pi \epsilon / k_{B} T$. In what follows, we use reduced units: we use the hard-sphere diameter $\sigma$ as our unit of length, and the thermal energy $k_{B} T$ as our unit of energy. 
Lebowitz and Penrose [4] were able to show that, in the limit $\lambda \rightarrow \infty$, the free energy per unit, the form of Eq. (1) is given exactly by

$$
f=\operatorname{M.C.F.}\left(f^{(0)}(\rho)+a \rho^{2}\right),
$$

where M.C.F.() denotes the maximal convex function not exceeding the function in parentheses, and $f^{(0)}(\rho)$ is the exact free energy per unit volume of hard spheres in a system with a number density $\rho$ (in particular, within the coexistence region it is a weighted mean of the free energies of the coexisting phases). Thus $f$ is given by the expression in parentheses except when the expression in parentheses is not convex, when it is given by the free energy along a straight line between two points of the curve such that with the straight line the function is rendered convex. When a straight line is required we are within the coexistence region of a firstorder phase transition.

In Fig. 1, we show the (almost [6]) exact phase diagram of hard spheres plus an infinitely long-range interaction. The $a<0$ half corresponds to the conventional van der Waals case. The negative $a \rho^{2}$ term in the free-energy density tends to make the function in parentheses nonconvex. This broadens the coexistence region of the existing fluid-crystal transition and, for $a<-5.55$, creates a first-order transition between two fluid phases: a vaporliquid transition. The fluid-crystal and vapor-liquid transitions meet at the triple point. The phase diagram of a simple atomic system, such as argon, has the same features as the $a<0$ part of Fig. 1 .

Along the $a=0$ line we have the phase behavior of hard spheres and for $a>0$ we have that of hard spheres

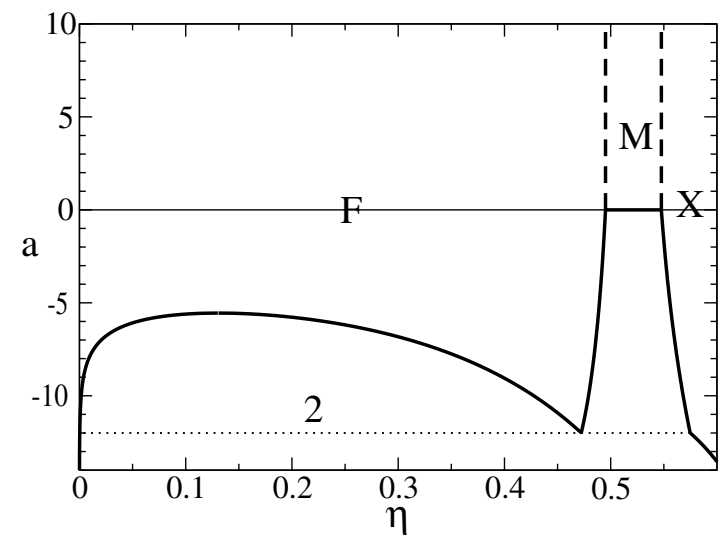

FIG. 1. The "exact" phase diagram for hard spheres with an infinitely long-ranged attraction or repulsion; in the $\eta$ - $a$ plane, where $\eta$ is the volume fraction occupied by the hard cores. The solid curves separate the 1 and 2 phase regions; the 2 phase region is marked by a 2 and the 1 phase fluid and crystal regions are marked by an F and an X, respectively. Microphase separation occurs between the vertical dashed lines, and is denoted by an M. The horizontal dotted line joins the three coexisting densities at the triple point. with a long-range repulsion [8]. For $a>0$ there is no fluid-crystal coexistence region as there is no plateau in the pressure versus density plot. Yet, the system does freeze: for densities below the continuation of the freezing density of the van der Waals fluid (the leftmost dashed curve), the system is a bulk fluid, while for densities above the continuation of the melting curve of the solid (the dashed curve on the right), the system is a bulk crystal.

We can see that the free energy of Eq. (3) implies this. $f^{(0)}$ is convex and, hence, adding a term that is quadratic in density with a positive coefficient, $a>0$, will not make it nonconvex. Thus, for $a>0, f$ is just given by the term in parentheses in Eq. (3).

In the hard-sphere fluid-crystal coexistence region, $f^{(0)}$ is a linear function of density. As $f$ has the additional $a \rho^{2}$ term it contains no straight segments, and hence there is no first-order transition. This is also obvious if one considers that the pressure $p=p^{(0)}+a \rho^{2}$, where $p^{(0)}$ is the pressure of hard spheres. In the hard-sphere coexistence region, $d p^{(0)} / d \rho=0$, but $d p / d \rho=2 a \rho>0$. In Fig. 1 we have drawn dashed curves to indicate the densities where $d p / d \rho$ is discontinuous.

Thus far, we have simply applied the Lebowitz-Penrose argument to the freezing transition of repulsive spheres. Next, we look in more detail at the nature of this unusual freezing transition. In particular, we consider what is happening in the potentially more relevant case that the range of the repulsion, $\lambda$, is large but finite. The key point to note about the potential of Eq. (1) is that it is characterized by two widely differing length scales: the hard-sphere interaction acts on a length scale $\sigma$ and the continuous repulsion extends over a length scale $\lambda \sigma \gg \sigma$.

At length scales of order $\sigma$, the hard-sphere interaction dominates and the system is structurally identical to a hard-sphere fluid (or solid). To see this, consider the force on a particle in a bulk phase, due to the long-range part of the interaction. The force tends to 0 as $\lambda \rightarrow \infty$ for two reasons: the pair energy of interaction is of order $\epsilon \lambda^{-3}$ but the pair force is a factor $\lambda$ smaller. Moreover, when adding the pair forces due to $\mathcal{O}\left(\rho \lambda^{3}\right)$ different interactions, the net force scales as $\left(\rho \lambda^{3}\right)^{1 / 2}$. Hence, in the limit $\lambda \rightarrow \infty$, the force on the particles in a bulk phase due to long-range interaction vanishes as $\lambda^{-5 / 2}$.

This implies that the fluid-to-crystal phase transition for hard spheres plus an infinitely long-range interaction (attractive or repulsive) starts off in exactly the same way as it does for hard spheres [9]. Hard spheres crystallize from the fluid via nucleation and growth $[10,11]$. As a typical crystal nucleus of a hard-sphere fluid contains only a few dozen to a few hundred particles [11], its free energy and dynamics are unaffected by the longrange interaction, as $\lambda$ is much larger than the size of the nucleus.

The differences between hard spheres and systems with long-range forces show up once nuclei have passed the 
nucleation barrier. In the absence of long-ranged forces, crystal nuclei grow and coalesce to form a single bulk crystalline phase, coexisting with a bulk fluid phase. The driving force for domain growth is provided by the hardsphere fluid-crystal interfacial free energy, $\gamma_{\mathrm{hs}}$ [12].

However, when $a>0$ we have seen that we do not have bulk phase coexistence. Let us consider a system in the fluid-crystal transition region, with domains of fluid and crystal intermixed on a length scale $l$. Specifically, consider the density to be a square-wave function of position along one direction, with a period $l$, and so a wave vector $q=2 \pi / l$. In the troughs the density is that in the fluid phase, $\rho_{F}$, and in the peaks, the density is that in the crystal phase, $\rho_{X}$. Thus the density modulation has a magnitude $\left(\rho_{X}-\rho_{F}\right)$. The free-energy change of creating the square-wave modulation costs an amount, per unit volume, of order $\sim \gamma_{\mathrm{hs}} q$, from the fluid-crystal interfaces. But it also changes the energy due to the long-range repulsion. Approximating this energy change by the change due to the first Fourier mode at wave vector $q$, and adding the interface term, we have

$$
f_{l} / k T \sim \gamma_{\mathrm{hs}} q+\left(\rho_{X}-\rho_{F}\right)^{2} \frac{a}{\left(1+q^{2} \lambda^{2}\right)^{2}} .
$$

The fraction is half the Fourier transform of the longrange part of the potential, Eq. (2). We neglected higher Fourier modes, despite the fact that the fluid-crystal interfaces are sharp on the length scale $l$. However, more detailed calculations yield the same behavior as Eq. (4) [14]. Anticipating that the length scale $l \ll \lambda$ we drop the 1 in the denominator. The two contributions to the surface free-energy density are comparable when

$$
q \sim\left(\frac{\left(\rho_{X}-\rho_{F}\right)^{2} a}{\gamma_{\mathrm{hs}}}\right)^{1 / 5} \lambda^{-4 / 5} .
$$

This happens when the domains have grown to a size $l \sim$ $\lambda^{4 / 5}$. For large $\lambda$, this is much larger than the hard-sphere diameter, yet smaller than $\lambda$ itself. For a long-range attraction, $a<0$, and both terms of the free energy, Eq. (4), decrease as $l$ increases. The minimum free energy is achieved as $l \rightarrow \infty$ : two coexisting bulk phases. However, for a long-range repulsion the two terms compete: the hard-sphere term favors large domains, but the long-range repulsion favors small domains. The equilibrium domain size is determined by this competition and given by Eq. (5): the long-range repulsion breaks up the coexisting bulk fluid and crystal phases of hard spheres into domains whose size scales as $\lambda^{4 / 5}$. Lebowitz and Penrose [4] appreciated that the bulk phases were broken up into "droplets or froth" but did not estimate the characteristic length scale. In the limit $\lambda \rightarrow \infty$, the size of domains is much less than $\lambda$ itself and hence the longrange repulsion sees a uniform density $\rho$, both inside and outside the coexistence region of hard spheres. That is why its contribution to the free-energy density is simply $a$ times the square of the mean density $\rho$.

The effect of long-range repulsion is then similar to that of surfactants: it breaks up bulk phase coexistence into microphase coexistence. In fact, this analogy becomes even more clear if we consider the effect of a long-range repulsion on the interfacial free energy. We start with a planar interface between coexisting fluid and crystalline phases of hard spheres, and add a long-range repulsion. We estimate the change in interfacial free energy, $\gamma_{l r}$, as simply the energy change in creating the interface due to the long-range repulsion, using the density profile of the interface in hard spheres. This density profile changes over from $\rho_{X}$ to $\rho_{F}$ over a region with a thickness of a few $\sigma$, and so for large $\lambda$ we may approximate the density profile by a step function. It is then easy to show that the change in interfacial free energy in a hard-sphere system with a long-ranged interaction potential of the form given by Eq. (2) is

$$
\gamma=\gamma_{\mathrm{hs}}-\frac{3}{2} a\left(\rho_{X}-\rho_{F}\right)^{2} \lambda .
$$

The key point to note is that, at fixed $a$, the contribution to the long-range contribution to the interfacial free energy is negative and scales as $\lambda$. When $a$ reaches $\lambda^{-1}\left(\rho_{X}-\rho_{F}\right)^{-2}$, the interfacial free energy becomes zero and bulk coexistence is not stable. A long-range repulsion destroys bulk coexistence at an integrated strength $a$ which varies as one over its range $\lambda$.

However, for a repulsion with a finite range, the interfacial free energy is positive over a range of values of $a$. Under those conditions, phase separation is still possible. The reduction in interfacial free energy scales with the square of the density gap, of order $10^{-2}$ here. This small prefactor implies that $a \lambda$ must be larger than $\mathcal{O}\left(10^{2}\right)$ in order to see microphase separation. For smaller values of $a \lambda$, bulk phase separation will remain, but the reduction in interfacial free energy will affect crystal nucleation. According to classical nucleation theory, the nucleation rate depends exponentially on (minus) the cube of the interfacial free energy [10]. As a consequence, it is strongly affected even by small variations in the interfacial free energy. In fact, experimentally it is well known that colloids with a soft repulsive interaction crystallize much more easily than hard-sphere colloids [15] and this observation is supported by recent simulations of crystal nucleation in suspensions of charged colloids [16]. We stress that this effect shows up only when the range of the repulsion is not much larger than the size of the nucleus. In the limit $\lambda \rightarrow \infty$, the initial nucleation rate is not affected by long-ranged repulsion.

The present paper focuses on the effect of long-ranged repulsion on the freezing of three-dimensional systems. However, the tendency of a long-range repulsion to turn bulk phase coexistence into microphase separation is general. For example, it also converts vapor-liquid 
coexistence into microphase separation [17], and there is much work on the effect of $r^{-3}$ repulsions in two dimensions $[18,19]$. The effect of long-ranged repulsions on nucleation rates has been studied in a somewhat different context, namely, that of nucleation near a critical point [20]. Long-range repulsive forces may also affect the scenario for two-dimensional melting. This may be relevant for the observation by Zahn et al. [21] of continuous two-stage melting in a quasi-2D system of magnetic colloids with a long-ranged $r^{-3}$ repulsion. As an $r^{-3}$ repulsion destroys macroscopic phase coexistence [19], the presence of this interaction may allow the appearance of a hexatic phase that would otherwise be preempted by a first-order freezing transition.

In summary, we have calculated the (almost) exact phase diagram of hard spheres with long-range attractions or repulsions, Fig. 1. For a long-range attraction the bulk phase behavior is qualitatively what we expect for a simple liquid such as argon, but the dynamics of the phase transition are anomalous. At a sufficiently large range of the attraction, the nucleation of the crystalline phase is indistinguishable from that of hard spheres. A long-range repulsion has a very different effect: it acts like a surfactant, decreasing the interfacial free energy [see Eq. (6)], eventually driving it to zero. Then, microphase separation replaces bulk phase separation. This conclusion is based on the analysis of Lebowitz and Penrose that becomes exact in the $\lambda \rightarrow \infty$ limit. In our analysis of systems with finite-ranged repulsions, we estimated how the repulsive potential affects the interfacial free energy and the length scale of microphase separation [Eq. (5)]. The transition from bulk fluid to bulk crystal proceeds via microphaseseparated phases and is continuous in the limit $\lambda \rightarrow \infty$. Surprisingly, although the transition is continuous, the crystal phase has to form via nucleation.

Experimental realizations of systems with long-ranged repulsion exist in two dimensions $[18,19]$. In three dimensions, it is possible to form Coulomb crystals of unscreened ions in a trap [22], but we are not aware of any detailed analysis of the liquid-solid transition in these systems. In suspensions of charged colloids, the electrostatic repulsions are screened, if not by added salt then by intervening macro ions themselves. Calculations of an interface in a model of charged proteins show that the electrostatic repulsion yields a negative contribution to the interfacial free energy [23]; the effect, while strong enough to enhance nucleation, does not lead to microphase separation.

We thank P. Warren for a careful reading of the manuscript. The work of the FOM Institute is part of the research program of FOM and is made possible by financial support from The Netherlands organization for Scientific Research (NWO).
[1] H. C. Longuet-Higgins and B. Widom, Mol. Phys. 8, 549 (1964).

[2] M. Kac, G. E. Uhlenbeck, and P. Hemmer, J. Math. Phys. (N.Y.) 4, 216-228 (1963).

[3] N. G. van Kampen, Phys. Rev. 135, A362 (1964).

[4] J. L. Lebowitz and O. Penrose, J. Math. Phys. (N.Y.) 7, 98 (1966).

[5] J. P. Hansen and I. R. McDonald, Theory of Simple Liquids (Academic Press, London, 1986), 2nd ed.

[6] For the fluid phase we use thermodynamic functions consistent with the pressure equation of state of Carnahan and Starling, and for the crystalline phase we use the fit to simulation data of Hall [7]. These expressions are both very accurate and so using them we have the almost exact thermodynamics of hard spheres.

[7] N. E. Carnahan and K. E. Starling, J. Chem. Phys. 51, 635 (1969); K. R. Hall, J. Chem. Phys. 57, 2252 (1972).

[8] W. G. Hoover and F. H. Ree, J. Chem. Phys. 49, 3609 (1968); P. G. Bolhuis, D. Frenkel, S.-C. Mau, and D. A. Huse, Nature (London) 388, 236 (1997).

[9] Not all phase transformations start with nucleation; some start with spinodal decomposition [10]. But this cannot occur here outside of the vapor-liquid transition envelope, as there the structure factor $S(q)>0 \forall q$.

[10] P. G. Debenedetti, Metastable Liquids (Princeton University Press, Princeton, 1996).

[11] S. Auer and D. Frenkel, Nature (London) 409, 1020 (2001).

[12] The fluid-crystal interface of hard spheres has been studied by Davidchack and Laird [13]. The interfacial free energy does depend on the orientation of the interface with respect to the crystal lattice, but it turns out that this dependence is relatively weak [13].

[13] R. L. Davidchack and B. B. Laird, Phys. Rev. Lett. 85, 4751 (2000).

[14] R. P. Sear and D. Frenkel (to be published).

[15] C. Smits, J. S. van Duijneveldt, J. K. G. Dhont, H. N.W. Lekkerkerker, and W. J. Briels, Phase Transit. 21, 157 (1990).

[16] S. Auer and D. Frenkel, J. Phys. Condens. Matter 14, 7667 (2002).

[17] R. P. Sear and W. M. Gelbart, J. Chem. Phys. 110, 4582 (1999); R. P. Sear, S.-W. Chung, G. Markovich, W. M. Gelbart, and J. R. Heath, Phys. Rev. E 59, R6255 (1999).

[18] M. Seul and D. Andelman, Science 267, 476 (1995).

[19] D. Andelman, F. Broçhard, and J.-F. Joanny, J. Chem. Phys. 86, 3673 (1987).

[20] D. Pini, G. Jialin, A. Parola, and L. Reatto, Chem. Phys. Lett. 327, 209 (2000).

[21] K. Zahn, R. Lenke, and G. Maret, Phys. Rev. Lett. 82, 2721 (1999).

[22] J. N. Tan, J. J. Bollinger, B. Jelenkovic, and D. J. Wineland, Phys. Rev. Lett. 75, 4198 (1995).

[23] R. P. Sear and P. B. Warren, J. Chem. Phys. 117, 8074 (2002). 